ISSN 0001-6012/2015/57/1/16-22 Acta Médica Costarricense, (ㅇ 2015 Colegio de Médicos y Cirujanos de Costa Rica

\title{
Original
}

\section{Caracterización farmacoterapéutica de los anticoagulantes orales utilizados en pacientes hospitalizados}
(Characterization of oral anticoagulant prescription in patients hospitalized (Costa Rica)

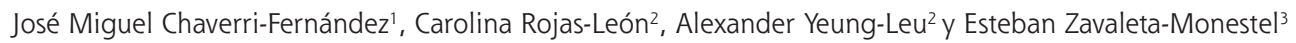

Trabajo realizado en el Hospital Clínica Bíblica, San José, Costa Rica.

Afiliación de los autores: 'Departamento de farmacología, toxicología y farmacodependencia de la Facultad de Farmacia de la Universidad de Costa Rica y Hospital Clínica Bíblica. 2Estudiantes de internado en Farmacia, Universidad de Ciencias Médicas. ${ }^{3}$ Farmacia Hospital Clínica Bíblica. San José, Costa Rica.

凶jose.chaverri@ucr.ac.cr

\section{Resumen}

Justificación: los anticoagulantes orales requieren una estricta farmacovigilancia; describir y analizar la utilización de estos medicamentos es importante para editar guías, recomendaciones y advertencias ante el uso de esta terapia, y así brindar al personal médico una herramienta de apoyo para su adecuada utilización.

Métodos: se seleccionaron pacientes a quienes durante su estancia hospitalaria se les prescribiera algún anticoagulante oral. Se recolectó la información específica que facilitara el análisis. Para validar lo recopilado se utilizaron las guías del Colegio Americano de Cirujanos Torácicos y las guías americanas y europeas de los colegios de cardiología.

Resultados: se recopiló la información de un total de 176 pacientes. Los especialistas que prescriben estos medicamentos son ortopedistas (40\%), internistas (14\%), cardiólogos (13\%) e intensivistas (11\%), para el tratamiento de tromboprofilaxis de accidente cerebrovascular, tromboprofilaxis postoperatoria, tratamiento del tromboembolismo pulmonar y de trombosis venosa profunda; el $84 \%$ de los pacientes que usó estos medicamentos, lo hizo en indicaciones aprobadas. Se reportó un $3,4 \%$ de sangrados.

Conclusión: la mayoría de las prescripciones se realizaron de acuerdo con lo establecido por las guías; a pesar de esto, existe una evidente incongruencia con respecto a los exámenes solicitados y su monitorización. La mayor proporción de los sangrados reportados se asoció a warfarina; aún así, seguirá siendo el estándar para el manejo de este tipo de pacientes, hasta que se apruebe tratamientos de reversión para los nuevos anticoagulantes y métodos adecuados para su monitorización, en los casos que se requiera.

Descriptores: anticoagulantes orales, farmacia clínica, trombosis, farmacovigilancia.

\section{Abstract}

Background: Oral anticoagulants require a strict pharmacovigilance; it is important to describe and analyze use of these drugs in order to draft guidelines, recommendations and warnings, and thus provide medical personnel with a tool for their adequate use.

Methods: Inpatients that received oral anticoagulants were selected and the specific information for this analysis was collected. The guidelines of the American College of Chest Physicians, as well as those of the American and European professional associations of cardiology were used. 
Results: Information from 176 patients was collected. The specialists that prescribe these drugs are orthopedists $(40 \%)$, internal medicine $(14 \%)$, cardiologists $(13 \%)$ and intensive care $(11 \%)$, for the treatment of stroke thromboprophylaxis, postoperative thromboprophylaxis, treatment of pulmonary embolism and deep vein thrombosis; $84 \%$ of the patients that used these drugs did so in approved prescriptions. Bleeding related to these drugs was reported in $3.4 \%$ of the cases.

Conclusion: Most prescriptions were administered according to the guidelines, despite this there is a clear inconsistency between tests ordered and their monitoring. Most bleeding reports were associated to warfarin, yet it remains the standard treatment for this kind of patients until reversion treatments for new oral anticoagulants and suitable methods for monitoring, when required, are approved.

Keywords: Oral anticoagulants, clinical pharmacy, thrombosis, pharmacovigilance.

Fecha recibido: 5 de febrero de 2014

Fecha aprobado: 18 de septiembre de 2014

Desde su introducción, los antagonistas de la vitamina $\mathrm{K}$ (AVK) han sido la base del tratamiento anticoagulante oral en múltiples indicaciones, siendo la warfarina (inhibidor de la síntesis de los factores de coagulación dependientes de la vitamina K) el medicamento con el que se ha desarrollado mayor experiencia clínica hasta el momento. Tras la introducción de los nuevos anticoagulantes orales (ACO), los cuales se orientan específicamente a inhibir factores de coagulación como el factor Xa (rivaroxaban) o IIa (trombina; dabigatran), el panorama ha cambiado. ${ }^{1}$

En la práctica clínica, la warfarina presenta importantes limitaciones que complican su uso. Posee un estrecho margen terapéutico, requiere pruebas periódicas que garanticen que el paciente está recibiendo la dosis adecuada para mantenerse anticoagulado (mediante el Índice Internacional Normalizado, INR, por sus siglas en inglés), y tiene múltiples interacciones medicamentosas y un lento inicio de acción. Mantener al paciente dentro del rango de dosis ideal, permite garantizar la eficacia y reducir al mínimo el riesgo de sangrado, principal y más importante efecto secundario de la warfarina., ${ }^{2,3}$

Los anticoagulantes orales como el dabigatran (inhibidor directo de la trombina o IIa) y el rivaroxaban (inhibidor del factor Xa) (Pradaxa ${ }^{\circledR}$ y Xarelto®, respectivamente), disponibles en el mercado costarricense desde diciembre de 2013, han demostrado, con algunos estudios clínicos (artículos publicados), ventajas sobre la warfarina en la prevención del accidente cerebrovascular de tipo isquémico, en pacientes con fibrilación atrial no valvular, en la prevención de trombosis venosa profunda(TVP)(postquirúrgica en cirugías de cadera y rodilla) y en su manejo terapéutico. ${ }^{4 \cdot 6}$

Existen notorios beneficios adicionales con estos fármacos, como el hecho de que alimentos específicos no generan reducciones sustanciales en sus efectos, además de la presencia de un menor índice de interacciones medicamentosas clínicamente relevantes. ${ }^{7}$ Estos anticoagulantes generan efectos consistentes con amplios márgenes terapéuticos en comparación con la warfarina, y no requieren la monitorización rutinaria de los tiempos de coagulación (su efecto es suficientemente predecible, por lo que se facilita su administración). ${ }^{7}$
A pesar de su aparente seguridad, no se cuenta hasta el momento con métodos de monitorización validados; si se deseara medir el efecto anticoagulante del dabigatran por alguna situación especial (prequirúrgico, sangrado activo, sobredosis, daño renal), ${ }^{8}$ el tiempo de tromboplastina parcial activado (TTPa; más sensible que el tiempo de Protrombina, TP) está indicado para aproximarlo. Otras pruebas que poseen una relación lineal con la concentración plasmática de dabigatran no se encuentran comercialmente disponibles. ${ }^{9}, 10$ En el caso del rivaroxaban, el TP (preferiblemente Neoplastin Plus®, Roche $)^{11}$ y el anti Xa cromogénico son los más adecuados para evaluar el grado de anticoagulación, debido a su mayor sensibilidad y su relación lineal con la concentración, aunque los resultados varían del reactivo y no son totalmente aceptados como pruebas válidas para la toma de decisiones.

Como es de esperarse, existe una relación entre la intensidad de la anticoagulación y el sangrado; a diferencia de la warfarina, los nuevos anticoagulantes aun no tienen antídotos específicos. Se ha planteado la suspensión inmediata del tratamiento junto a la observación, debido a su corta vida media, además de la utilización de carbón activado 2 y 8 horas posteriores a la última administración de dabigatran y rivaroxaban, respectivamente, 0 la posibilidad de utilizar Factor VIla recombinante o complejo concentrado de protrombina / complejo concentrado de protrombina activado (CCP/CCPa)., $3,8,9,11,12$

Según las guías más recientes del Colegio Americano de Cirujanos Torácicos (ACCP), las publicaciones realizadas en su revista oficial (CHEST) y la Sociedad Europea de Cardiología (ESC), la terapia anticoagulante sigue siendo el componente principal para la prevención y el tratamiento de la TVP y el tromboembolismo, el cual incluye el tromboembolismo pulmonar (TEP) y la protección contra el accidente cerebrovascular isquémico secundario a fibrilación atrial (FA): ${ }^{13}$ estos últimos tienen mayor riesgo de sufrir un evento tromboembólico y se estratifican de acuerdo con un sistema de puntaje, según la presencia de factores de riesgo $\left(\mathrm{CHADS}_{2} \mathrm{O}\right.$ $\mathrm{CHA}_{2} \mathrm{DS}_{2}$-VASc). ${ }^{14,15}$

Para brindar una mayor claridad de la "situación actual", se presenta una breve comparación entre los anticoagulantes orales disponibles en Costa Rica, con énfasis en sus características 


\begin{tabular}{|c|c|c|c|}
\hline Característica & Warfarina & Dabigatran & Rivaroxaban \\
\hline Blanco de acción & $\begin{array}{l}\text { Factores Vitamina K } \\
\text { dependientes }\end{array}$ & Factor Ila (Trombina) & Factor Xa \\
\hline Biodisponibilidad & $100 \%$ & $3-7 \%$ & $80 \%$ \\
\hline $\begin{array}{l}\text { Tiempo máximo } \\
\text { concentraciones }\end{array}$ & 4-5 días & $1-2 \mathrm{~h}$ & $2-3 \mathrm{~h}$ \\
\hline Vida media & $40 \mathrm{~h}$ & $8-17 \mathrm{~h}$ & $5-9 h$ \\
\hline Eliminación renal & No aplica & $80 \%$ & $33 \%$ \\
\hline Interacciones & CYP 2C9/ 1A2/3A4 & Glicoproteína-P & CYP 3A4 / Glicoproteína-P \\
\hline Monitorización & TP , INR & Ninguno validado & Ninguno validado \\
\hline Antídoto & Vitamina K & Ninguno validado & Ninguno validado \\
\hline Indicaciones aprobadas & $\begin{array}{c}\text { Prevención de } \\
\text { tromboembolismo venoso } \\
\text { (TEV) y tratamiento } \\
\text { o complicaciones } \\
\text { tromboembólicas asociadas } \\
\text { con FA y prevención secundaria } \\
\text { postinfarto agudo del } \\
\text { miocardio }\end{array}$ & $\begin{array}{c}\text { Prevención de } \\
\text { tromboembolismo } \\
\text { venoso postquirúrgico } \\
\text { (cirugía de cadera o } \\
\text { rodilla), prevención } \\
\text { de embolismo } \\
\text { sistémico y accidente } \\
\text { cerebrovascular (ACV) en } \\
\text { pacientes con FA }\end{array}$ & $\begin{array}{c}\text { Prevención de TEV } \\
\text { postquirúrgico en cirugías } \\
\text { de cadera o rodilla, } \\
\text { prevención de embolismo } \\
\text { sistémico y ACV en pacientes } \\
\text { con FA, tratamiento para } \\
\text { TVP aguda y prevención } \\
\text { de recurrencias de } \\
\text { tromboembolismo venoso }\end{array}$ \\
\hline
\end{tabular}

farmacocinéticas, farmacodinámicas e indicaciones aprobadas internacionalmente, así como sus limitaciones. (Cuadro1)

Esta investigación pretende, a partir de un estudio observacional, describir y analizar la utilización de los anticoagulantes orales de reciente introducción en Costa Rica. Se procura suministrar información sobre estos medicamentos de reciente ingreso al mercado y que todavía precisan estudios y una estricta y adecuada farmacovigilancia, validando así su seguridad y efectividad en el tiempo. Dichos datos facilitarán también la elaboración de protocolos o lineamientos, que brinden al personal médico una herramienta de apoyo para la adecuada utilización y toma de decisiones asociadas al uso de estos productos.

\section{Métodos}

Se realizó un estudio observacional de tipo retrospectivo, tomando como población a todos los pacientes a quienes, durante su estancia hospitalaria (enero 2012 - junio 2013) se les prescribiera algún anticoagulante oral de los registrados para su uso en el país (rivaroxaban, dabigatran, warfarina).

Los datos relacionados con la población en estudio fueron obtenidos mediante consultas electrónicas al Sistema Electrónico del Centro Médico (SIGH) o el expediente clínico del paciente (electrónico o impreso, según se necesitara).

Se recolectó los datos que facilitaran el análisis del uso de los medicamentos descritos. Cada paciente debía cumplir con los siguientes criterios de inclusión: mayor de 18 años de edad; uso de un anticoagulante oral de los registrados a la fecha de este análisis en Costa Rica (warfarina, dabigatran y rivaroxaban), durante o posterior a su internamiento; al menos 2 días de hospitalización, y ausencia de contraindicaciones absolutas para el uso de cada anticoagulante mencionado. Los criterios de exclusión fueron: que los pacientes fueran mujeres embarazadas o pacientes fallecidos, cuya muerte no estuviera relacionada con el uso de ACO.

Algunas de las variables por contemplar y recolectar de cada expediente, que permitan un análisis a profundidad fueron: anticoagulante prescrito, tipo de médico que prescribe según especialidad, indicación en la que se está utilizando el medicamento, dosis (correcta o no, según indicación), ajuste de dosis (si fue requerido), frecuencia, duración del tratamiento, presencia de interacciones clínicamente significativas, métodos utilizados para la monitorización del anticoagulante durante el internamiento (INR, TTPa, TP, hematocrito, hemoglobina, entre otros y sus posibles variaciones durante el internamiento), efectos secundarios asociados al efecto terapéutico de los productos, patologías concomitantes, uso de otros medicamentos que afecten la coagulación de uso paralelo, y duración del tratamiento proyectado.

Para validar lo recopilado con respecto al tratamiento anticoagulante prescrito, se utilizan las guías del Colegio Americano de Cirujanos Torácicos, ${ }^{16-24}$ las guías americanas (ACC) $)^{25,26}$ y europeas de los colegios de cardiología (ESC). 2,27 En estas se detalla sobre el uso de este tipo de medicamentos, dosis, indicaciones aprobadas y demás detalles relacionados, junto con la información científica utilizada por las agencias internacionales para la aprobación de estos medicamentos en sus mercados específicos. 
Caracterización de anticoagulantes orales / Chaverri-Fernández et al.

\begin{tabular}{|ccc|}
\hline $\begin{array}{c}\text { Cuadro 2. Porcentaje de uso de anticoagulantes } \\
\text { orales, según diagnóstico en pacientes internados en } \\
\text { el Hospital ClínicaBíblica }\end{array}$ \\
\hline $\begin{array}{c}\text { Indicación del } \\
\text { anticoagulante }\end{array}$ & Porcentaje & Total de casos \\
\hline $\begin{array}{c}\text { Tromboprofilaxis } \\
\text { postoperatoria de cadera } \\
\text { Profilaxis accidente } \\
\text { cerebrovascular }\end{array}$ & 27 & 43 \\
$\begin{array}{c}\text { Tromboprofilaxis } \\
\text { postoperatoria de rodilla }\end{array}$ & 19 & 40 \\
$\begin{array}{c}\text { Trombosis venosa profunda } \\
\text { Tromboembolismo } \\
\text { pulmonar }\end{array}$ & 12 & 20 \\
$\begin{array}{c}\text { Otra indicación no } \\
\text { aprobada }\end{array}$ & 4 & 11 \\
\hline Total & 100 & 176 \\
\hline
\end{tabular}

Con respecto a los efectos adversos reportados, y con el fin de conocer acerca de la seguridad en general de los anticoagulantes orales, se llevó también un registro de la frecuencia de aparición y métodos establecidos para su control.

Para el análisis de los datos se utilizó los programas SPSS y MS Excel, en sus últimas versiones disponibles.

Para el desarrollo de este estudio se cuenta con la aprobación de la Dirección de Educación Médica e Investigaciones del Hospital Clínica Bíblica, a través de la Academia Strachan, la cual garantiza, junto con los investigadores, el manejo adecuado, ético y la confidencialidad de los datos de los pacientes incluidos en el estudio. Adicionalmente, se cuenta con la aprobación del Comité Ético Científico de la Universidad de Ciencias Médicas.

\section{Resultados}

Durante el periodo de estudio se recopiló la información perteneciente a 176 pacientes (52\% hombres - $48 \%$ mujeres), con una edad promedio de 65 años (rangos entre 45-65 años). Su estancia intrahospitalaria promedio fue de 4 días (máximas y mínimas de 25 y 2 días, respectivamente). Del total de pacientes, el 51\% (90 casos) utilizó rivaroxaban, el 23\% (40 casos), warfarina y el $26 \%$ (46 casos) dabigatran, como anticoagulante.

Los especialistas que prescriben mayoritariamente estos medicamentos son ortopedistas (40\%), internistas (14\%), cardiólogos (13\%) e intensivistas (11\%), aunque otros profesionales estuvieron también involucrados en la utilización de tales fármacos.

Los diferentes diagnósticos a partir de los cuales se prescribieron los anticoagulantes en estudio, incluyeron fibrilación atrial (según la estratificación de riesgo realizada por el tratante para tromboprofilaxis de accidente cerebrovascular), tromboprofilaxis postoperatoria (cirugía de rodilla y cadera), tratamiento del tromboembolismo pulmonar, tratamiento de trombosis venosa profunda y otras indicaciones no aprobadas (tromboprofilaxis postoperatoria de tibia, codo, ortejos, clavícula, tobillo y muñeca, politraumatismo, ángor inestable, laparotomía exploratoria y sepsis). La proporción en la que se usó los anticoagulantes, según indicación, se detalla en el Cuadro 2.

Del total de pacientes que utilizó un ACO para la prevención del accidente cerebrovascular, 20 (50\%) utilizaron dabigatran, $10(25 \%)$, rivaroxaban y 10 (25\%), warfarina.

Para la indicación de tromboprofilaxis postoperatoria de rodilla, se empleó en 11 ocasiones (32\%) dabigatran, en $22(65 \%)$, rivaroxaban y en una ocasión (3\%), warfarina. En tromboprolaxis postoperatoria de cadera se utilizó en $8(19 \%)$ oportunidades dabigatran, y en 35 (81\%), rivaroxaban.

Para el manejo de la trombosis venosa profunda se usó en 4 (20\%) ocasiones rivaroxaban y en 16 (80\%), warfarina. Finalmente, para el abordaje del tromboembolismo pulmonar se usó en 2 (18\%) ocasiones rivaroxaban y en 9 (82\%), warfarina.

Un 16\% de los pacientes que empleó estos medicamentos lo hizo en indicaciones aún no aprobadas por las guías internacionales (tromboprofilaxis postoperatoria de tibia, codo, ortejos, clavícula, tobillo y muñeca, politraumatismo, ángor inestable, laparotomía exploratoria y sepsis). Es preciso aclarar que las guías internacionales sugieren recomendaciones de uso para el tratante, elaboradas por asociaciones de alguna especialidad o un ente técnico, sin embargo, no son entes reguladores que puedan oficializar indicaciones. En cada caso de los mencionados existió un único diagnóstico del paciente que justificó la utilización del anticoagulante.

La dosificación según la indicación para la cual fue utilizado el anticoagulante oral, fue correcta (en los casos con duración reportada) en un 50\% (20) de los casos de los pacientes con fibrilación atrial (tromboprofilaxis de accidente cerebrovascular), en un 86\% (37) de los casos de tromboprofilaxis de TVP (cirugía de cadera), en un $27 \%$ (3) de los casos de tromboembolismo pulmonar, y en un 5\% (1) de los casos de trombosis venosa profunda.

La duración del tratamiento fue adecuada en un 40\% de los casos para warfarina, en un $63 \%$ de los casos para dabigatran y en un $81 \%$ de los casos para rivaroxaban. Todo lo anterior, según lo sugerido por las guías internacionales.

La cuantificación del aclaramiento renal fue estimada en un $60 \%$ de las ocasiones. En 6 oportunidades fue inferior a $30 \mathrm{ml} / \mathrm{min}$, y en 12 casos el aclaramiento estuvo entre $30 \mathrm{y}$ $50 \mathrm{ml} / \mathrm{min}$, circunstancia que sugiere ajustar la dosis, según el producto utilizado (rivaroxaban o dabigatran), realizando un balance riesgo - beneficio. No se detectó modificaciones en los casos en los cuales eran sugeridos tales ajustes, ni se reportó problemas relacionados con tal circunstancia. 


\begin{tabular}{|c|c|c|c|}
\hline \multicolumn{4}{|c|}{$\begin{array}{l}\text { Cuadro3. Porcentajes de análisis de laboratorio } \\
\text { utilizados en la monitorización de pacientes } \\
\text { internados, según anticoagulante oral en el Hospital } \\
\text { Clínica Bíblica }\end{array}$} \\
\hline & Warfarina & Dabigatran & Rivaroxaban \\
\hline INR & $34 \%$ & $17 \%$ & $36 \%$ \\
\hline $\begin{array}{l}\text { Tiempo de } \\
\text { protrombina } \\
\text { (TP) }\end{array}$ & $35 \%$ & $17 \%$ & $36 \%$ \\
\hline $\begin{array}{l}\text { Tiempo } \\
\text { parcial de } \\
\text { tromboplastina } \\
\text { activada (TTPa) }\end{array}$ & $29 \%$ & $17 \%$ & $35 \%$ \\
\hline Dímero D & $11 \%$ & $3 \%$ & $6 \%$ \\
\hline Antifactor Xa & $7 \%$ & - & $2 \%$ \\
\hline Antitrombina III & $6 \%$ & - & $1 \%$ \\
\hline Proteína C & $6 \%$ & - & $1 \%$ \\
\hline Proteína S & $6 \%$ & - & $1 \%$ \\
\hline Fibrinógeno & $2 \%$ & - & $1 \%$ \\
\hline Ninguno & $3 \%$ & $28 \%$ & $53 \%$ \\
\hline
\end{tabular}

En regímenes en donde se utilizó warfarina, los ajustes de dosificación no fueron necesarios. Con frecuencia se comprobó el empleo de fármacos antiagregantes plaquetarios concomitantes, según algunas necesidades fisiopatológicas del paciente. En un 24\% (43 casos) de las ocasiones se prescribió concomitantemente aspirina, clopidogrel y enoxaparina (ya sean estos medicamentos solos, o en combinación, además de los anticoagulantes orales analizados). La combinación más frecuente fue con enoxaparina, en un $84 \%$ de las ocasiones, seguida por aspirina en un $14 \%$ de y clopidogrel en una proporción mucho menor (12\%).

Respecto al sangrado, se reportó en un 3,4\% de los casos estudiados, que equivale a un total de 6 sangrados, distribuidos de la siguiente manera: 2 reportados con dabigatran, 1 con rivaroxaban y 3 con warfarina. Los eventos se abordaron mediante trasfusión sanguínea, suspensión del tratamiento, o sin reporte de ningún cambio a la estrategia terapéutica, respectivamente.

En un $80 \%$ de las ocasiones se utilizó el hematocrito y la hemoglobina como parámetros para la monitorización de la seguridad de los anticoagulantes (riesgo de sangrado de la anticoagulación como efecto secundario), con cambios significativos (según lo que la evidencia reporta como cambios importantes por tomar en cuenta en el hematocrito y hemoglobina $)^{5,8,9,11}$ en el $29 \%$ y el $21 \%$ de las ocasiones, respectivamente. Es pertinente mencionar que se cuantificó la presencia de sangrado mediante otra metodología, ni hubo cambios en las estrategias medicamentosas establecidas. Diferentes indicadores hematológicos se usaron para la monitorización de la terapia anticoagulante. En el Cuadro 3 se representa la proporción de uso de tales métodos, clasificados por anticoagulante.

En el $88 \%$ de los internamientos estudiados no se realizó cambios en el tratamiento anticoagulante oral en estudio. Cuando el médico tratante efectuó cambios o modificaciones, respondían a cambios en la dosificación (50\%), cambios en el anticoagulante (35\%) o modificación de la vía de administración dirigida hacia otro producto anticoagulante (15\%). Se presentó interacciones medicamentosas clínicamente significativas, en un $73 \%$ de las ocasiones que sugerían vigilancia del tratamiento, mas no necesariamente su suspensión.

\section{Discusión}

De acuerdo con los resultados de la investigación, es posible presumir que en la mayoría de las intervenciones el médico tratante tiene conocimiento de cuáles son las principales circunstancias en las cuales se utilizan los anticoagulantes orales. Es decir, mayoritariamente, las prescripciones de anticoagulantes se realizaron de acuerdo con lo establecido en la bibliografía. ${ }^{16}$

Es importante resaltar que las indicaciones por las que suelen prescribirse los anticoagulantes orales (ACO) en el Hospital Clínica Bíblica, son la prevención del accidente cerebrovascular (según la estratificación de riesgo establecida por el tratante) y la profilaxis de trombosis venosa profunda, posterior a una cirugía de rodilla o cadera. Lo anterior está directamente relacionado con el hecho de que las especialidades que prescribieron en la mayoría de las ocasiones el anticoagulante oral, fueron Ortopedia y Traumatología. Al hablar de las indicaciones no aprobadas, cabe destacar que este hecho no implica necesariamente un mal abordaje por parte del prescriptor, sino que hasta la fecha se carece de estudios que demuestren la utilidad, seguridad y eficacia del ACO en las indicaciones específicas, o que las autoridades respectivas aun no aprueban su uso. En cuanto a la duración del tratamiento, las guías y estudios clínicos recientes definen una determinada para todas las indicaciones, excepto en la prevención de accidente cerebrovascular (que precisa tratamiento continuo). A pesar de que en la mayoría de las ocasiones la duración fue la correcta, se evidencia que se requiere mejorar la divulgación de los datos científicos que respaldan una correcta duración y sus beneficios. ${ }^{2,16-27}$

El INR es un valor de laboratorio indispensable en caso de decidir si se debe realizar un ajuste de dosis en el empleo de warfarina, con el fin degarantizar su seguridad y efectividad (el valor ideal INR se ajusta de acuerdo con el diagnóstico, y oscila usualmente entre de $2-3$, y un $65 \%$ se encuentra dentro del valor ideal). No existe a la fecha un examen de laboratorio validado que permita monitorizar al rivaroxaban o dabigatran, lo que evidentemente limita la verificación de la adherencia por parte del paciente y, por supuesto, la seguridad asociada al riesgo de sangrado. ${ }^{29}$

Los datos evaluados revelan que existe una clara incongruencia con respecto a los exámenes solicitados y la monitorización de la respuesta medicamentosa, en la mayoría 
Caracterización de anticoagulantes orales / Chaverri-Fernández et al.

de los casos en los que se prescribió warfarina (en función de ajustar su INR o valorar la seguridad del tratamiento). ${ }^{30}$ Debe mencionarse que los nuevos anticoagulantes orales no requieren ajuste de la dosis sobre la base de una prueba de coagulación específica, solo lo precisan si el aclaramiento renal está afectado. ${ }^{27}$

Conviene mencionar que la medición de antifactor Xa cromogénico utilizado en 2 ocasiones para el rivaroxaban, se relaciona de forma directa con la concentración del fármaco y su efectividad, estimando así potencialmente el efecto anticoagulante; a pesar de esto, la prueba todavía no está validada para ese fin. ${ }^{27}$

Con respecto a la incidencia de interacciones medicamentosas clínicamente relevantes, debe recordarse que la warfarina es metabolizada por las isoenzimas del citocromo P-450: CYP2C9, CYP2C8, CYP2C18, CYP2C19, CYP1A2 y CYP3A4. Los nuevos anticoagulantes orales, por su lado, se ven afectados por la P-glicoproteína (bomba de eflujo) en el caso del dabigatran y rivaroxaban, y por la isoenzima del citocromo P-450 CYP3A4, para rivaroxaban. Esto en definitiva influye en la aparición de un número importante de potenciales interacciones medicamentosas. ${ }^{29,31,32}$

La mayor proporción de los sangrados reportados se asoció a warfarina, aspecto esperable debido a lo difícil de garantizar una dosis segura, en función de las múltiples variables que afectan su metabolismo y concentración plasmática. ${ }^{7,31,32}$

Aunque las estrategias para revertir el sangrado por warfarina están definidas (administración de un agente de reversión específico oral o intravenoso: vitamina K), la bibliografía actual proporciona poca orientación en cuanto a la reversión adecuada con los nuevos anticoagulantes, ya que carecen de antídotos específicos. Existe evidencia, en estudios con animales in vitro, de la posible utilidad del complejo de protrombinasa activado (CCPa), o la administración de factores de coagulación especíicicos, o plasma fresco. Algunos antídotos, en los casos de rivaroxaban y apixaban, están en desarrollo.6,10,33,34

En el caso específico de sangrado por rivaroxaban, se sugiere la administración de factor VII activado, elaborado por técnicas recombinantes (rFVIIa) o CCPa. Para dabigatran es preferible emplear rFVIIa. ${ }^{11}$ El rivaroxaban y dabigatran poseen una vida media relativamente corta, y la interrupción del tratamiento se recomienda en caso de eventos de sangrado y el establecimiento de protocolos de rutina, para el manejo de hemorragias. ${ }^{28}$

La aparición de los nuevos anticoagulantes ha despertado el interés del personal médico del Hospital Clínica Bíblica, en tanto resulta evidente que se requiere nuevos métodos para monitorizar todos los ACO, siendo estos medicamentos, productos que potencialmente llegarían a desplazar las terapias estándares en el corto o mediano plazo.

Por su parte, la warfarina seguirá siendo un estándar para el manejo de este tipo de pacientes, hasta que se apruebe tratamientos de reversión para los nuevos anticoagulantes, y métodos adecuados para su monitorización. En definitiva, para el manejo ambulatorio, los nuevos productos (ACO) podrían mejorar la calidad de vida del paciente, su adherencia en el tiempo e incluso su efectividad.

Conflictos de interés: los autores declaran que no existe ningún conflicto de interés que afecte la investigación.

\section{Referencias}

1. Höchtl T, Huber K. New anticoagulants for the prevention of stroke in atrial fibrillation. Fund Clin Pharmacol. 2012;26:47-53.

2. Perk J, De Backer G, Gohlke H, Graham I, Reiner Z, Verschuren M, et al. European Guidelines on cardiovascular disease prevention in clinical practice. Eur Heart J. 2012;33:1635-1701.

3. Lader E, Martin N, Cohen G, Meyer M, Reiter P, Dimova A, et al. Warfarin therapeutic monitoring: is $70 \%$ time in the therapeutic range the best we can do? J Clin Pharm Ther. 2012;37:375-377.

4. Kanagasabapathy P, Chowdary P, Gatt A. Alternatives to Warfarin-The Next Generation of Anticoagulants. Cardiovasc Ther. 2011;29: e80-e88.

5. Pérez A, Eraso LH, Merli GJ. Implications of new anticoagulants in primary practice. Int J Clin Pract. 2013;67:139-156.

6. Smythe MA, Fanikos J, Gulseth MP, Wittkowsky AK, Spinler SA, Dager WE, Nutescu EA. Rivaroxaban: Practical considerations for ensuring safety and efficacy. Pharmacotherapy. 2013;33:1223-45.

7. Walenga JM, Adiguzel C. Drug and dietary interactions of the new and emerging oral anticoagulants. Int J Clin Pract. 2010;64:956-67.

8. Eby, C. Novel anticoagulants and laboratory testing. Int J Lab Hematol. 2013;35:262-268.

9. Baglin T. The role of the laboratory in treatment with new oral anticoagulants. J Thromb Haemost. 2013;11(Suppl. 1):122-128

10. Sarah S. The pharmacology and therapeutic use of dabigatran etexilate. J Clin Pharmacol. 2013;53:1-13.

11. Peacock WF, Gearhart MM, Mills RM. Emergency management of bleeding associated with old and new oral anticoagulants. Clin Cardiol. 2012;35:730737.

12. Harris K, Mant J. Potential impact of new oral anticoagulants on the management of atrial fibrillation-related stroke in primary care. Int J Clin Pract. 2013;67:647-655.

13. Tripodi A, Palareti G. New anticoagulant drugs for treatment of venous thromboembolism and stroke prevention in atrial fibrillation. J Intern Med. 2012;271:554-565.

14. Verheugt FW. Advances in stroke prevention in atrial fibrillation: enhanced risk stratification combined with the newer oral anticoagulants. Clin Cardiol. 2013;36:313-322.

15. Turpie AGG. Rivaroxaban for the prevention and treatment of venous thromboembolism.Fund Clin Pharmacol. 2012;26:33-38.

16. Eikelboom, John M., Hirsh J, Frederick A. Spencer, Baglin T, and Weitz J. Antiplatelet drugs: Antithrombotic therapy and prevention of thrombosis. Chest. 2012;141:89-119.

17. Per Olav V. Lincoff M. Gore J. Gutterman D, Sonnenberg Coello P. Elie A. Lansberg M. Primary and secondary prevention of cardiovascular disease. Chest. 2012;141:637-668

18. Whitlock R. Sun J. Fremes S. Rubens F. Antithrombotic and thrombolytic therapy for valvular disease. Antithrombotic therapy and prevention of thrombosis. Chest. 2012;141: 576-600.

19. Lansberg M. O.Donnel M. Khatri P. Lang E. Nguyen-Huynh M. Schwartz N. Antithrombotic and thrombolytic therapy for ischemic stroke. Antithrombotic therapy and prevention of thrombosis. Chest. 2012;141:601-636.

20. You J. Singer D. Howard P. Lane D. Eckman M. Fang M. Hylek E. Schulman S. Hughes M. Spencer F. Manning W. Halperin J. Lip G. Antithrombotic therapy 
for atrial fibrillation. Antithrombotic therapy and prevention of thrombosis. Chest. 2012;141:531-575

21. Kearon C, Akl E, Comerota A, Prandoni P, Bounameaux H, Goldhaber $\mathrm{S}$, et al. Antithrombotic therapy for VTE disease: Antithrombotic therapy and prevention of thrombosis, 9th ed: American College of Chest Physicians evidence-based clinica practice guidelines. Chest. 2012;141:419S-494S.

22. Douketis J, Spyropoulos A, Spencer A, Mayr M, Jaffer A, Eckman M, et al. Perioperative management of antithrombotic therapy. Antithrombotic therapy and prevention of thrombosis. Chest. 2012;141;326S-350S.

23. Falck-Ytter Y. Francis C. Johanson N. Curley C. Dahl E. Schulman S. Ortel T. Pauker S. Cohwell Jr C. Prevention of VTE in orthopedic surgery patients. Antithrombotic therapy and prevention of thrombosis. Chest. 2012;141:278S-325S

24. Ageno W, Gallus AS, Wittkowsky A, Crowther M, Hylek EM, Palareti G. Oral Anticoagulant Therapy therapy and prevention of thrombosis. Chest. 2012;141:44S-88S.

25. Anderson J. Halperin J. Albert N. Brindis R. Curtis L. Hochman J. Kovacs R, et al. Management of Patients with Atrial Fibrillation: A Report of the American College of Cardiology/American Heart Association Task Force on Practice Guidelines Circulation. 2013;127:1916-1926.

26. Wann S, Curtis A, Ellenbogen K, Estes M, Ezekovitz M, Jackman W, et al. Focused Update on the Management of Patients With Atrial Fibrillation (Update on Dabigatran)." American College of Cardiology. 2011. [Internet]. 14 Agosto. 2013. [citado 20 Diciembre 2010]. http://circ.ahajournals.org/ content/123/1/104.
27. Camm J. Lip G. De Caterina R. Savelieva I. Atar D. Hohnloser S. Hindricks G. Kirchhof P. Focused update of the ESC Guidelines for the management of atrial fibrillation. Eur Heart J. 2012;33:2719-2747.

28. Turpie AG, Kreutz R, Llau J, Norrving B, Haas S. Management consensus guidance for the use of rivaroxaban -an oral, direct factor Xa inhibitor. J Thromb Haemost. 2012;108:876-886.

29. Schulman S. Advantages and limitations of the new anticoagulants. J Intern Med. 2014;275:1-11.

30. Rose AJ, Berlowitz DR, Miller DR, Hylek EM, Ozonoff A, Zhao S, Reisman JI, Ash AS. INR targets and site-level anticoagulation control: results from the Veterans Affairs Study to Improve Anticoagulation (VARIA). J Thromb Haemost. 2012;10:590-595.

31. Bungard T; YakiwchuK; Foisy M; Brocklebank C. Drug interactions involving warfarin: Practice tool and practical management tips. Can Pharm J. 2011;144: 21-25.

32. Maddison J, Somogyi AA, Jensen BP, James HM, Gentgall M, Rolan PE. The pharmacokinetics and pharmacodynamics of single dose $(\mathrm{R})$ and $(\mathrm{S})$-warfarin administered separately and together: relationship to VKORC1 genotype. J Clin Pharmacol. 2013;75:208-216.

33. Kazmi RS, Lwaleed BA. New anticoagulants: how to deal with treatment failure and bleeding complications. J Clin Pharmacol. 2011;72:593-603.

34. Eerenberg ES, Kamphuisen PW, Sijpkens MK, Meijers JC, Buller HR, Levi M. Reversal of rivaroxaban and dabigatran by prothrombin complex concentrate: A randomized, placebo-controlled, crossover study in healthy subjects. Circulation. 2011;124:1573-1579. 